\title{
Interplanetary fast forward shocks and energetic storm particle events above $1.5 \mathrm{MeV}$
}

\author{
K. Huttunen-Heikinmaa and E. Valtonen \\ Space Research Laboratory, Department of Physics and Astronomy, University of Turku, 20014 Turku, Finland
}

Received: 9 September 2008 - Revised: 17 December 2008 - Accepted: 23 January 2009 - Published: 17 February 2009

\begin{abstract}
Interplanetary (IP) shock passages are usually identified by abrupt changes in the plasma parameters, but sometimes they are also associated with energetic storm particles (ESPs). The maximum observed energies of ESPs usually reach a few MeVs per nucleon and occasionally even a few hundred MeVs per nucleon. We have carried out a statistical study of ESP events observed by SOHO/ERNE above $1.5 \mathrm{MeV}$ during the seven-year period between May 1996 and April 2003. In the first stage, we gathered a comprehensive database of IP shock candidates using several readymade shock lists. We defined a qualitative classification for the ESP signals and studied their association with fast forward shocks. We present a survey of the overall statistics of ESP associations with fast forward shocks and the yearly amount of the shocks and associated ESP events during the 7 -year study period. Our most important findings are that only $40 \%$ of the observed interplanetary fast forward shocks accelerate ESPs to energies greater than $1.5 \mathrm{MeV}$ and that the high-energy ESP-effectiveness of the fast forward shocks has a solar cycle dependence. The yearly ESP-effectiveness varied from 11\%, in May 1996-April 1997 ( activity minimum), to 53\% in May 2000-April 2001 ( activity maximum). We also performed a quantitative analysis of the proton power law spectra at the time of the shock passage. We found that the average spectral index of ESPs was -3.6 with the standard deviation of the distribution of 1.3. The ESP events had significantly larger power law factors than the reference spectra, calculated every day at a certain time for comparison.
\end{abstract}

Keywords. Solar physics, astrophysics, and astronomy (Energetic particles)

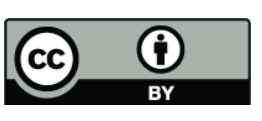

Correspondence to:

$\mathrm{K}$. Huttunen-Heikinmaa

(kahehu@utu.fi)

\section{Introduction}

Coronal mass ejections (CMEs) and their interplanetary counterparts (ICMEs) are the primary drivers of interplanetary (IP) shocks (e.g. Lindsay et al., 1994; Berdichevsky et al., 2000, 2001). Other possible drivers of IP shocks are stream interaction regions (SIR) which form when a fast solar wind stream overtakes a slow stream (Jian et al., 2006, and references therein). Investigation of IP shocks is important because of their role in particle acceleration (Reames, 1999) and as a cause of geomagnetic storms (Richardson et al., 2001; Zhang et al., 2007a,b). Shock passages are usually identified as abrupt changes in the solar wind plasma parameters, but sometimes they are also associated with energetic storm particles (ESPs) (Bryant et al., 1962; Rao et al., 1967). Acceleration of ESPs in ICME-driven (transient) interplanetary shocks has been studied by many authors, e.g. Tsurutani and Lin (1985), Kallenrode (1996), Lario et al. (2003), Ho et al. (2003), and Channok et al. (2005). The time development of proton and ion energy spectra, and the event time profiles were investigated by van Nes et al. (1984), Meyer et al. (1993), Desai et al. (2004), Kallenrode (1997), and Luhmann and Mann (2007). In particular ESPs associated with fast forward transient interplanetary shocks have been studied statistically by Lario et al. (2005). A recent overview of ESP observations has been given by Cohen (2006). Energetic storm particles may provide information not only on the shock acceleration processes, but also on the structure and evolution of the shock (Cane, 1995). Furthermore, since the intensities of ESPs often start to rise several hours before the shock passage, ESP observations can possibly be used as precursors of approaching shocks/ICMEs potentially causing geoeffects (Valtonen et al., 2005).

We have studied energetic storm particle events observed by SOHO/ERNE during the seven-year period between May 1996 and April 2003. We have gathered a comprehensive database of interplanetary shock candidates using several

Published by Copernicus Publications on behalf of the European Geosciences Union. 
Table 1. Database source catalogs. Study period: May 1996-April 2003.

\begin{tabular}{|c|c|c|c|c|c|c|c|c|c|}
\hline \multirow[b]{2}{*}{ Catalog } & \multirow[b]{2}{*}{ Cover period ${ }^{\mathrm{a}}$} & \multicolumn{2}{|c|}{ Entries $^{b}$} & \multicolumn{2}{|c|}{ This database $\mathrm{c}^{\mathrm{c}}$} & \multicolumn{2}{|c|}{ Covered $^{\mathrm{d}}$} & \multicolumn{2}{|c|}{ Unique $^{\mathrm{e}}$} \\
\hline & & $\mathrm{ALL}^{\mathrm{f}}$ & hq-FF' & ALL & hq-FF & ALL & hq-FF & ALL & hq-FF \\
\hline ACE list ${ }^{\mathrm{h}}$ & $2.9 .97-(30.4 .03)$ & 296 & 209 & 544 & 275 & $54 \%$ & $76 \%$ & 21 & 3 \\
\hline Berdichevsky et al. list $\mathrm{t}^{\mathrm{i}}$ & $(1.5 .96)-17.2 .03$ & 379 & 251 & 595 & 299 & $64 \%$ & $84 \%$ & 110 & 12 \\
\hline CELIAS shockspotter ${ }^{\mathrm{j}}$ & $26.9 .96-(30.4 .03)^{\mathrm{k}}$ & 193 & 166 & 572 & 283 & $34 \%$ & $59 \%$ & 17 & 1 \\
\hline D. Larson's list ${ }^{1}$ & $(1.5 .96)-25.6 .98$ & 84 & 44 & 130 & 66 & $65 \%$ & $67 \%$ & 34 & 2 \\
\hline Howard and Tappin (2005) & $1.1 .98-(30.4 .03)$ & 309 & 214 & 517 & 256 & $60 \%$ & $84 \%$ & 25 & 3 \\
\hline J. Kasper's ACE list ${ }^{\mathrm{m}}$ & $4.3 .98-24.12 .02$ & 155 & 150 & 481 & 246 & $32 \%$ & $61 \%$ & 2 & 1 \\
\hline J. Kasper's WIND list ${ }^{\mathrm{m}}$ & $16.6 .96-(30.4 .03)$ & 236 & 197 & 605 & 302 & $39 \%$ & $65 \%$ & 50 & 19 \\
\hline Database of the present work & $1.5 .96-30.4 .03$ & 606 & 302 & 606 & 302 & $100 \%$ & $100 \%$ & 4 & 1 \\
\hline
\end{tabular}

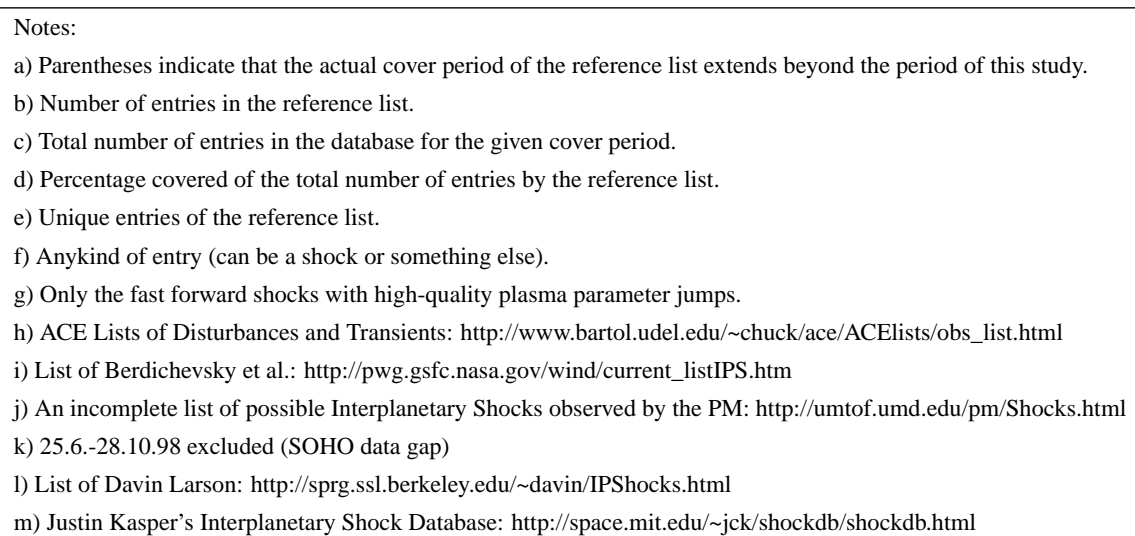

ready-made shock lists and searched for associated ESP events from the ERNE observations. In this paper, we present the results of a statistical study of the ESP associations of fast forward (FF) shocks regardless of their drivers. The achieved statistics will be used as the baseline in a follow-up study of the driver associations and their significance for the ESP characteristics, and will be presented in a separate paper.

In this paper we first deduce the magnetohydrodynamic (MHD) modes of the collected shock candidates (fast, slow, forward, or reverse), and then define a qualitative quick-look classification of the ESP signals associated with the identified FF shocks. In Sect. 2 we describe the method of shock mode identification and present statistics of IP shocks during the study period. In Sect. 3 we briefly review the availability of the ERNE particle data for the shock passage periods. The ESP signal classification is presented in Sect. 4 and some examples of ESP events are given. In Sect. 4 we also present a survey of the overall statistics of ESP associations with fast forward shocks and the yearly amount of FF shocks and associated ESP events during the 7-year study period. In Sect. 5 we investigate the ESP energy spectra at the time of the shock passages and compare them with random background spectra. Section 6 includes discussion and conclusions.

\section{Interplanetary shocks}

Interplanetary shocks are the basis of this study. Therefore, the list of shocks was compiled as comprehensive as possible. This was achieved by merging six different shock lists available on the internet (see url addresses in Table 1) and the list of Howard and Tappin (2005). For completeness, every single entry of the source lists was added in the database, even though almost all the lists contained candidates that were not shocks according to the criteria applied in this work (see below). In addition, four new shocks were identified in the vicinity of the listed candidates. The total number of shock candidate entries in the database reached 606. For 32\% (193) of those there is a CELIAS timing available. For the shock passage timings of this study, the times reported by the CELIAS Shockspotter (see Table 1) are the most accurate, because ERNE and CELIAS are on-board the same spacecraft. The time periods covered by various lists and the number of referenced entries are given in Table 1.

The shock candidates were classified by using solar wind and magnetic field data from WIND, ACE and SOHO spacecraft. The interplanetary magnetic field, $\boldsymbol{B}$, is observed by the WIND/MFI (Lepping et al., 1995) and ACE/MAG (Smith et al., 1998) instruments. The solar wind speed, $V_{\mathrm{sw}}$, proton temperature, $T_{p}$, and proton number density, $n_{p}$, are observed by the WIND/3DP (Lin et al., 1995), WIND/SWE 


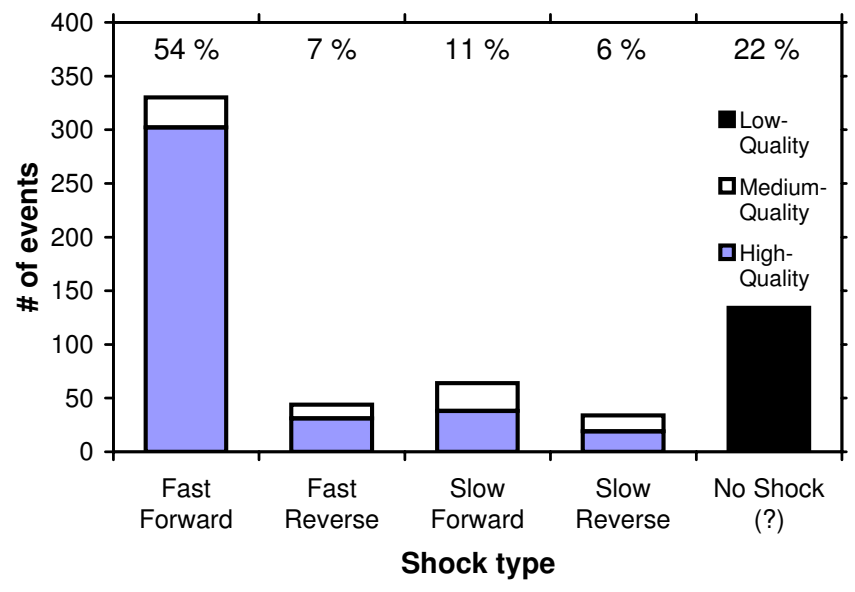

Fig. 1. The shock types of the database entries (606). The quality rating refers to the (subjective) reliability of the shock type identification.

(Ogilvie et al., 1995), ACE/SWEPAM (McComas et al., 1998) and SOHO/CELIAS (Hovestadt et al., 1995) instruments. WIND and ACE data plots were acquired from the Coordinated Data Analysis Web (http://cdaweb.gsfc.nasa. gov/cdaweb/istp_public/) and SOHO/CELIAS data plots from the CELIAS Shockspotter list web page.

The magnetohydrodynamic modes of the shock candidates were deduced by inspecting the behavior of the solar wind plasma parameters across the boundary. It is expected that for a fast forward (FF) shock the values of $B, V_{\mathrm{sw}}, T_{p}$ and $n_{p}$ increase as the spacecraft moves from the upstream region of the shock to the downstream region; for a fast reverse (FR) shock, only $V_{\mathrm{sw}}$ increases, and all the other parameters decrease; for a slow forward (SF) shock, only $B$ decreases, and all the others increase; and for a slow reverse (SR) shock, $B$ and $V_{\text {sw }}$ increase while $T_{p}$ and $n_{p}$ decrease (e.g. Echer et al., 2003).

The reliability of the MHD mode identification was also ranked. The candidates with unambiguous sudden changes in the plasma parameters gained "high-quality" ranking (390 cases). Essentially all the candidates that were found in the Justin Kasper's database (see Table 1) were regarded as reliable identifications. Hence, it seems that these plasma data had already been inspected very carefully. If the plasma parameter jumps were less pronounced (e.g., smoother), "medium-quality" was given (82). In cases when the behavior of the plasma parameters was undetermined or the candidate did not even look like a shock according to the criteria defined above, "low-quality" was selected (134). From all the database entries, 54\% (330) were fast forward shocks, $7 \%$ (44) fast reverse shocks, $11 \%$ (64) slow forward shocks, $6 \%$ (34) slow reverse shocks, and 22\% (134) something else. Therefore, a vast majority of the shock candidates were fast forward shocks. On the other hand, a notable fraction of the entries were judged not to be shocks at all, but rather

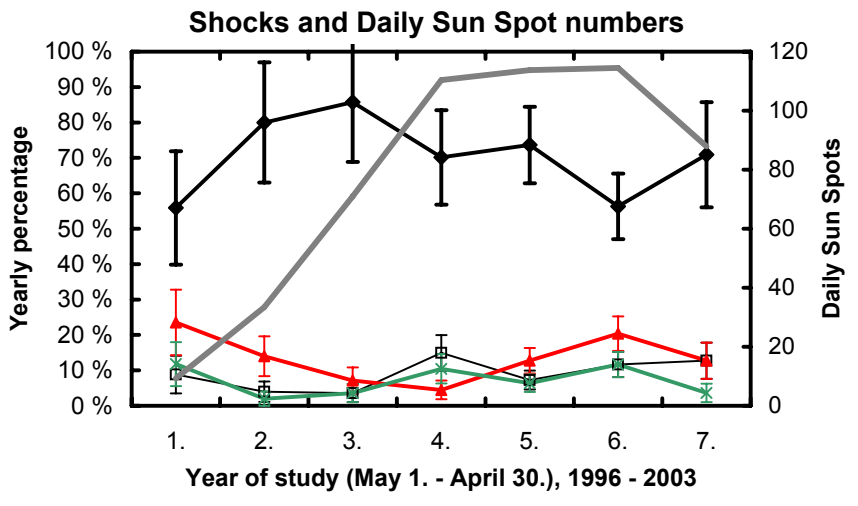

Fig. 2. Yearly percentages of different shock types and daily sunspot numbers. The yearly averages of daily sunspot numbers are presented as gray line without symbols. The share of fast forward shocks is presented with thick black line with diamonds. Fast reverse shocks: thin black line with open squares. Slow forward shocks: thick red line with triangles. Slow reverse shocks: thick green line with crosses.

other kind of discontinuities or even pure false identifications. Considering only "high-quality" plasma parameter jumps, the fraction of the fast forward shocks rises to $77 \%$ (302), while $8 \%$ (31) are fast reverse shocks, $10 \%$ (38) slow forward shocks, and 5\% (19) slow reverse shocks. The statistics of the deduced shock types are presented in Fig. 1.

The yearly percentages of the observed shock types during the seven years of this study are plotted in Fig. 2. To illustrate the development of solar cycle 23 , the yearly averages of daily sun spot numbers are also presented with the scale on the right. The daily sun spot numbers were aquired from the National Geophysical Data Center (ftp://ftp.ngdc.noaa.gov/ STP/SOLAR_DATA/SUNSPOT_NUMBERS/). Figure 2 might indicate that FF shocks are observed slightly more frequently in respect to the other shock types during the rising activity phase compared to other activity phases. However, as the statistical error bars show, the statistics of this study are not sufficient for firm conclusions. Regarding the error bars of FR, SF, and SR shocks, it should be noted that the yearly amounts of these shocks were small, generally $\lesssim 10$ year. Also, due to the limited time period of seven years this study does not tell anything of the relative shock occurrence frequencies during the declining phase of the solar cycle.

\section{Energetic particles}

For the ESP observations, 1-min SOHO/ERNE (Torsti et al., 1995) data were used. During the study period, there were some notable data gaps, and some of the very large particle events of the solar cycle 23 had corrupted data. The availability of ERNE data for the occurrence times of the shock candidates was divided in four groups. For 89\% (538) of all 


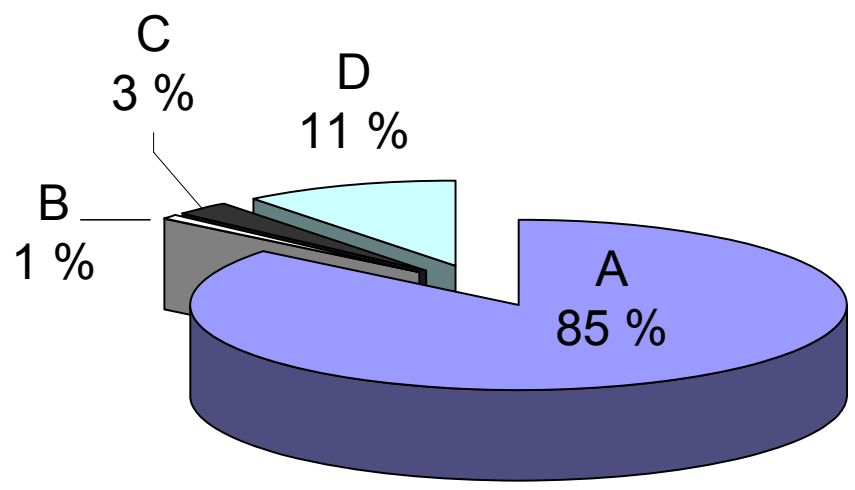

Fig. 3. ERNE data availability for the fast forward shocks (330). (A) data available for the shock passage period (a few hours before and after the reported shock arrival) and also for the time period of a few days before the shock passage, (B) data available for the shock passage period, but data gap before the passage period, (C) corrupted data, and (D) complete data gap.

the database entries data were available for the shock passage period (a few hours before and after the reported shock arrival) and also for a time period of a few days before the shock passage. For $1 \%(8)$ data were available for the shock passage period, but there was a data gap just before the passage period. For $2 \%$ (11) data were corrupted, and for $8 \%$ (49) there was a complete data gap. For fast forward shocks, the focus of this study, the ERNE data availability is presented in Fig. 3.

\section{Quick-look ESP signals}

It is useful to make a "quick-look" classification of the ESP signals associated with fast forward IP shock passages. In this study, intensity enhancements at several energy channels with no velocity dispersion in the vicinity of the shock passage are considered as ESP event candidates. Possible ESP signals can occur over the galactic cosmic ray background at high energies or be superposed on a Solar Energetic Particle (SEP) event. Using the ERNE on-board analysed proton data (1.5-130 MeV; 20 energy channels), the quick-look ESP signals were categorized into four classes.

There are "clearly" ESPs, when the intensity enhancements are temporally close to the shock passage and/or intensities are noticeably peaked. If the temporal relationship is less reliable or the enhancement is gradual, there are "probably" ESPs. If only a small intensity enhancement is seen at some limited energy range, the ESP observation is "ambiguous". Obviously, there are "no" ESPs, if no enhancement is detected at any energy channel. This classification is of course subjective to some extent.

Examples of ESP event candidates are presented in Fig. 4. Shock arrivals and driver candidates are marked in the panels for convenience. The timings of Magnetic Clouds (MC), In- terplanetary Coronal Mass Ejections (ICMEs), and Stream Interaction Regions (SIRs) are adapted from http://lepmfi. gsfc.nasa.gov/mfi/mag_cloud_pub1.html, Cane and Richardson (2003), and Jian et al. (2006), respectively.

Figure 4, panel (a) shows a "clear" ESP event of 18 May 2002 (shock arrival time 19:29 UT). The shock was driven by a magnetic cloud. This case represents rather common features, such as a preceding SEP event that is actually not a well-behaved smooth text-book-case, clearly peaked ESPs with the maximum temporally very close to the shock passage time, multiple intensity enhancements at several energy channels, and the front boundary of the driver cloud visible in the particle data as a drop in the intetensity curves. Panel (b) presents another typical "clear" ESP event of 10 April 1997 (12:58 UT). In this case, the shock seems to be SIR-driven, although the SIR is followed by an ICME. Panel (c) shows a "clear" ESP event of 19 Apri1 2002 (07:58 UT), which is related to an ICME-driven shock. The shock has apparently overcome the end boundary of an earlier ICME. This case is also an example in which the ESP event is more pronounced at higher particle energies. Panel (d) presents an interesting "clear" ESP event of 30 December 1997 (01:18 UT). It is particularly interesting, because there is no evidence for a primary SEP event and the intensity enhancement starts almost immediately after the shock passage.

Panel (e) of Fig. 4 shows a case, in which "probably" ESPs have been observed. The shock was observed on 20 February 2000 (20:56 UT) and the presumed ESP event occurred in the sheath region approximately peaking at the front boundary of the driver ICME. Panel (f) presents another case of "probably" ESPs. The ICME-driven shock was observed on 17 April 2002 (10:13 UT). The uncertainty of ESP observation comes from the fact that the shock passage occurs at the same time as an another SEP event commences. The presumed ESP event stands out because it starts earlier at low energies than the SEP event at higher energies. It also peaks at the time of the shock passage. The SEP-related rise of intensities is also seen later at lower energies, as one would expect due to the velocity dispersion.

Panels (g) and (h) show examples of cases that were settled to exhibit "ambiguous" ESPs. The shock in panel (g) was observed on 10 January 2002 (15:52 UT) and was most probably SIR-driven. There is a tiny intensity enhancement at the low energy channels right at the shock passage time, which may indicate the existence of ESPs. Panel (h) presents the shock on 29 July 2002 (12:44 UT), which has no driver candidate in the used catalogs. In addition to a slight intensity enhancement at low-energy channels, a clear decrement of intensity is observed right after the shock passage.

Figure 5 presents the statistics of the quick-look signals. It was found that $29 \%$ (85) of the fast forward shocks are "clearly" associated with high-energy ESPs ( $>1.5 \mathrm{MeV}$ protons), and 13\% (39) "probably" associated. Very weak signals were found for $12 \%$ (34) of the cases, and $46 \%$ (137) of the fast forward shocks had no associated high-energy 

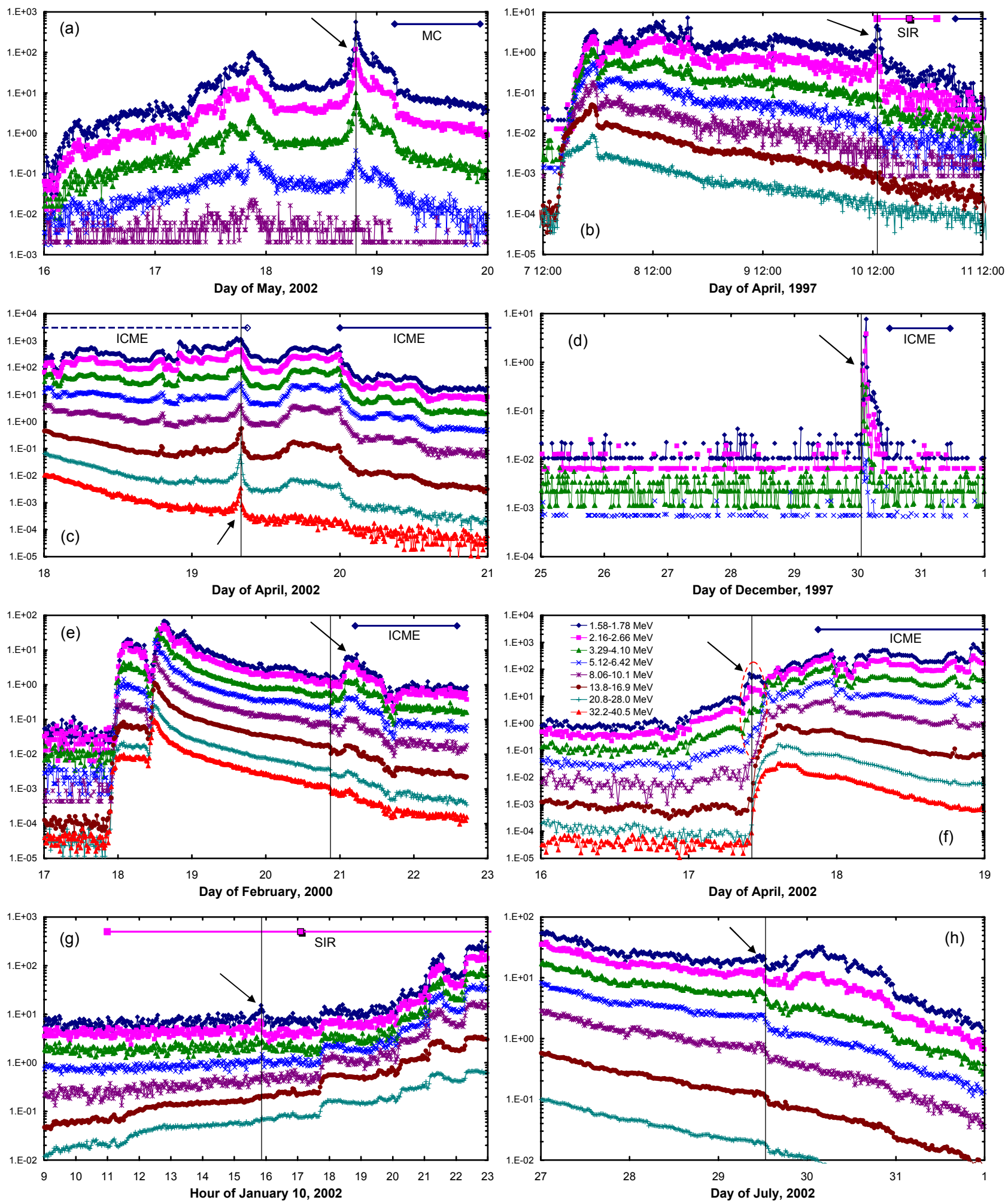

Fig. 4. Examples of observed ESP events. The curves in each panel represent ERNE proton intensities $\left[\left(\mathrm{cm}^{2} \mathrm{srs} \mathrm{MeV}^{-1}\right]\right.$ at selected energy channels. The energy ranges of the plotted channels are given in panel (f). The arrows indicate the oberved ESPs and the vertical lines indicate the fast forward shock passage times (from CELIAS). The possible identified drivers of the shocks are marked with horizontal lines: $\mathrm{MC}=$ Magnetic Cloud, SIR = Stream Interaction Region, and ICME = Interplanetary Coronal Mass Ejection. The events in panels $(\mathbf{a}-$ d) exhibit "clearly" ESPs, panels (e-f) "probably" ESPs, and (g-h) "ambiguous" ESPs. See text for details. 


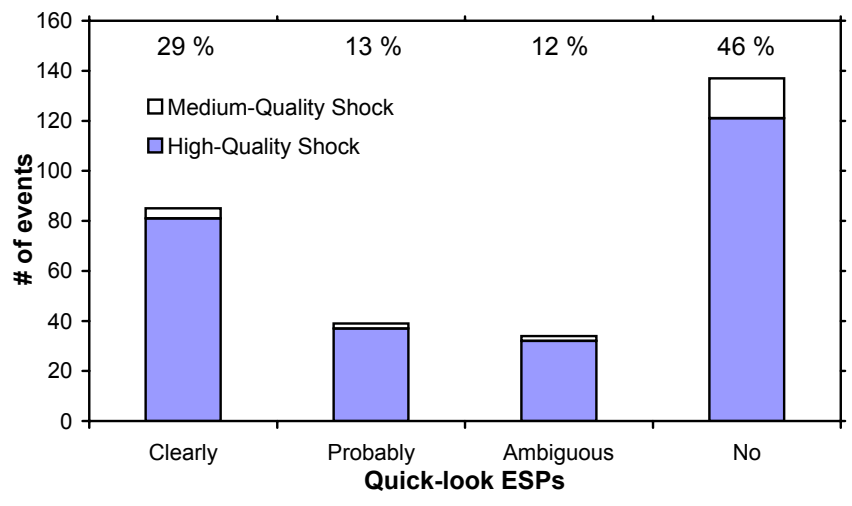

Fig. 5. Quick-look ESP signals for the fast forward shocks (295). The shock quality ratings refer to the (subjective) reliability of the shock mode identification. The shocks with no available ERNE data are excluded ("D" in Fig. 3).

ESPs. These percentages are for the fast forward shocks with medium- and high-quality plasma parameter jumps. When only the high-quality fast forward shocks are taken into account, the percentages remain basically the same: $30 \%(81)$, $14 \%$ (37), $12 \%$ (32), and $44 \%$ (121), respectively. It is notable that the fraction of the medium-quality fast forward shocks is larger in the "no ESPs" group than in the "clearly ESPs" group (Fig. 5). Table 2 lists all the fast forward shocks during the study period that possibly have at least some degree of association with high-energy ESPs.

The intensity measurements may include randomly some small enhancements, which are unrelated to shock passages. Therefore, it is plausible that the weak particle signals of the "ambiguous" group can not be regarded as reliable ESP signals. Interpretation of the group with probable association is more problematic. For that reason, it is difficult to give an accurate answer to the simple question of how large fraction of the fast forward shocks are associated with high-energy ESPs.

On one hand, the nature of all the particle enhancements can not be precisely defined. On the other hand, there are differences in the reliability of the shock mode identification. The question of high-energy ESP-effectiveness of interplanetary fast forward shocks can be approached in various ways. The most intuitive data set separations to positive and negative ESP signals are listed in Table 3. The first column of the table identifies the used quick-look ESP signals (Fig. 5) for the positive and negative ESP occurrence. The second column shows the reliability of fast forward shock identification. The percentages of positive and negative ESP signals are presented in columns 3 and 4, respectively. The bottom row of columns 3 and 4 gives the average percentages of the positive and negative ESP signals. Based on the statistics shown in Table 3, it can be stated that roughly $40 \%$ of the interplanetary fast forward shocks accelerate ESPs to energies greater than $1.5 \mathrm{MeV}$.

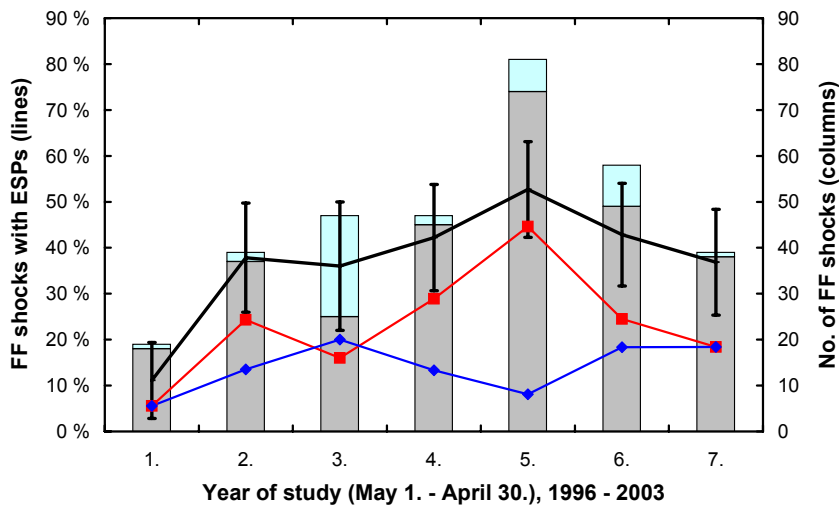

Fig. 6. Yearly amounts of interplanetary fast forward shocks (columns), and the fraction of those associated with high energy ESPs (lines). The percentages of ESP association are calculated for the grey columns, which represent the fast forward shocks with available ERNE data. Red squares represent the fraction of shocks "clearly" associated with ESPs and blue diamonds "probably" associated with ESPs. Black thick line with statistical error bars shows the sum of red squares and blue diamonds.

Yearly amount of the fast forward shocks (medium and high quality) during the seven study years is presented in Fig. 6 as columns. The gray-shaded parts of the columns indicate the cases with available ERNE data. The lines in Fig. 6 show the fraction of fast forward shocks that were associated with high energy ESPs. The percentages of ESP association were calculated for the shocks with available ERNE data. Red squares represent the fraction of shocks "clearly" associated with ESPs, and blue diamonds "probably" associated with ESPs. Black thick line with statistical error bars shows the total share of fast forward shocks associated with the two categories of ESP events.

The solar cycle variation is clearly seen in the yearly amount of fast forward shocks. During May 2000 April 2001 (column 5) roughly four times more fast forward shocks were observed than during May 1996-April 1997 (column 1). Interestingly, similar solar cycle variation is also seen in the high-energy ESP-effectiveness of the fast forward shocks. In Fig. 6, the black thick line basically follows the trend of the columns, if also the shocks without ERNE data are taken into account. When the high-energy ESPeffectiveness is divided into sub-groups of "clearly ESPs" and "probably ESPs", we can see that the "probably" group does not have any significant trend during the study period, whereas the "clearly" group follows the trend of the gray-shaded columns. The third year of study (May 1998April 1999) seems to be somewhat contradictory. During a large fraction of this time period there was an interruption in SOHO operation. Therefore the ERNE data coverage for the observed fast forward shocks is only $53 \%$. Thus, on one hand, the sample of fast forward shocks with ERNE data is smaller for the third year than for the second or the fourth 
Table 2. Fast Forward shock passages associated with high-energy ESPs from May 1996 to April 2003.

\begin{tabular}{|c|c|c|c|c|c|c|c|c|}
\hline Passage time $^{\mathrm{a}}$ & $S / C^{b}$ & $\mathrm{ESPs}^{\mathrm{c}}$ & Passage time & $\mathrm{S} / \mathrm{C}$ & ESPs & Passage time & $\mathrm{S} / \mathrm{C}$ & ESPs \\
\hline 1996/06/18 22:35 & $\mathrm{W}$ & A & 1999/09/22 11:45 & A W & $\mathrm{C}$ & 2001/05/08 09:31 & A W & A \\
\hline 1996/07/28 12:14 & $\mathrm{W}$ & A & $1999 / 10 / 2101: 34$ & S A W & $\mathrm{C}$ & 2001/05/12 09:24 & A W & $\mathrm{C}$ \\
\hline 1996/08/12 22:11 & $\mathrm{W}$ & A & $1999 / 11 / 21 \quad 16: 15$ & A W & $\mathrm{C}$ & $2001 / 05 / 2714: 17$ & S A W & $\mathrm{A}$ \\
\hline 1996/08/16 07:45 & W & A & $2000 / 01 / 11$ 13:40 & A S W & $\mathrm{C}$ & $2001 / 06 / 1801: 53$ & S A W & $\mathrm{C}$ \\
\hline 1996/08/22 13:10 & $\mathrm{W}$ & A & $2000 / 01 / 2200: 22$ & A S & $\mathrm{C}$ & $2001 / 08 / 05$ 11:55 & A W & $\mathrm{P}$ \\
\hline 1996/12/02 10:00 & $\mathrm{W}$ & $\mathrm{P}$ & $2000 / 01 / 2713: 56$ & A S W & A & 2001/09/14 01:08 & S A W & $\mathrm{C}$ \\
\hline 1997/02/09 12:43 & S W & A & $2000 / 02 / 1102: 12$ & A S W & $\mathrm{P}$ & $2001 / 09 / 2519: 51$ & S A W & $\mathrm{C}$ \\
\hline $1997 / 04 / 1012: 58$ & S W & $\mathrm{C}$ & $2000 / 02 / 1123: 18$ & A S W & $\mathrm{C}$ & $2001 / 09 / 29$ 09:03 & S A W & $\mathrm{C}$ \\
\hline 1997/05/15 00:55 & S W & $\mathrm{C}$ & $2000 / 02 / 2020: 45$ & A S W & $\mathrm{P}$ & 2001/09/30 18:41 & S A W & $\mathrm{P}$ \\
\hline 1997/05/26 09:09 & $\mathrm{W}$ & A & 2000/04/06 16:01 & S A W & $\mathrm{C}$ & 2001/10/01 21:30 & A W & A \\
\hline 1997/09/21 03:40 & $\mathrm{A} \mathrm{W}$ & $\mathrm{C}$ & 2000/04/24 08:51 & A W & $\mathrm{C}$ & 2001/10/03 08:06 & A & $\mathrm{P}$ \\
\hline 1997/10/01 00:20 & S A & $\mathrm{C}$ & 2000/05/12 17:13* & A W & $\mathrm{P}$ & 2001/10/08 12:21 & A W & A \\
\hline 1997/10/10 02:30 & A W & $\mathrm{C}$ & 2000/06/08 08:41 & A S W & $\mathrm{C}$ & $2001 / 10 / 11$ 16:13 & S A W & $\mathrm{C}$ \\
\hline 1997/10/10 15:30 & A W & $\mathrm{C}$ & $2000 / 06 / 11$ 12:42 & A W & $\mathrm{C}$ & $2001 / 10 / 21$ 16:05 & S A W & $\mathrm{C}$ \\
\hline 1997/10/24 10:20 & S A W & $\mathrm{P}$ & $2000 / 06 / 23 \quad 12: 26$ & A S W & $\mathrm{C}$ & $2001 / 10 / 2802: 33$ & S A W & $\mathrm{C}$ \\
\hline 1997/11/01 05:40 & A W & A & $2000 / 07 / 1005: 55$ & S A & $\mathrm{C}$ & 2001/11/06 01:20 & S A W & $\mathrm{C}$ \\
\hline 1997/11/06 22:02 & A S W & $\mathrm{P}$ & $2000 / 07 / 1111: 22$ & A & A & $2001 / 11 / 19$ 17:34 & S A W & A \\
\hline 1997/11/09 09:52 & A W & $\mathrm{C}$ & 2000/07/13 09:01 & A S W & $\mathrm{C}$ & $2001 / 11 / 2404: 54$ & $\mathrm{~W}$ & $\mathrm{C}$ \\
\hline 1997/11/09 22:00 & A W & $\mathrm{P}$ & 2000/07/13 09:19 & A S W & $\mathrm{C}$ & $2001 / 11 / 2405: 33$ & S A W & $\mathrm{C}$ \\
\hline 1997/11/22 08:55 & S A W & $\mathrm{C}$ & $2000 / 07 / 14$ 15:00 & A S W & $\mathrm{C}$ & $2001 / 12 / 21 \quad 13: 40$ & A W & $\mathrm{P}$ \\
\hline 1997/11/30 07:05 & A W & A & $2000 / 07 / 15$ 14:15 & A S W & $\mathrm{C}$ & $2001 / 12 / 2904: 47$ & A S W & $\mathrm{P}$ \\
\hline 1997/12/10 04:24 & S W & A & $2000 / 07 / 19$ 14:48 & A S W & $\mathrm{C}$ & $2001 / 12 / 3019: 31$ & A S W & $\mathrm{P}$ \\
\hline $1997 / 12 / 3001: 13$ & W S A & $\mathrm{C}$ & $2000 / 07 / 2617: 54$ & A S W & $\mathrm{P}$ & $2002 / 01 / 10 \quad 15: 45$ & A S W & A \\
\hline 1998/01/06 13:19 & S W A & $\mathrm{C}$ & $2000 / 07 / 2805: 42$ & A S W & $\mathrm{C}$ & 2002/01/19 04:10 & A & $\mathrm{C}$ \\
\hline 1998/01/28 15:45 & A W S & $\mathrm{P}$ & 2000/07/28 09:09 & A S W & $\mathrm{C}$ & $2002 / 01 / 3120: 40$ & A S W & $\mathrm{C}$ \\
\hline 1998/04/23 17:14 & S A W & $\mathrm{P}$ & 2000/08/11 18:10 & A S W & $\mathrm{C}$ & $2002 / 02 / 1701: 56$ & S A W & $\mathrm{A}$ \\
\hline 1998/04/30 08:43 & A W & $\mathrm{P}$ & $2000 / 08 / 1421: 36$ & A S W & $\mathrm{C}$ & $2002 / 03 / 18$ 12:33 & S A W & $\mathrm{P}$ \\
\hline 1998/05/01 21:15 & S W A & $\mathrm{C}$ & 2000/09/06 16:13 & A S W & $\mathrm{C}$ & 2002/03/20 13:05 & S A W & $\mathrm{C}$ \\
\hline 1998/05/04 02:03 & A W S & $\mathrm{C}$ & $2000 / 09 / 15$ 03:59 & A S W & $\mathrm{P}$ & $2002 / 03 / 23$ 10:47 & S A W & $\mathrm{P}$ \\
\hline 1998/05/04 02:29* & A W S & $\mathrm{C}$ & $2000 / 09 / 17$ 16:57 & A W & $\mathrm{C}$ & $2002 / 03 / 2500: 57$ & A S W & $\mathrm{A}$ \\
\hline 1998/05/08 09:20 & W A & $\mathrm{P}$ & $2000 / 10 / 05$ 02:36 & S A W & $\mathrm{C}$ & 2002/04/17 10:13 & S A W & $\mathrm{P}$ \\
\hline $1998 / 06 / 13 \quad 18: 25$ & S A W & $\mathrm{P}$ & $2000 / 10 / 1221: 36$ & S A W & $\mathrm{C}$ & $2002 / 04 / 1907: 58$ & S A W & $\mathrm{C}$ \\
\hline 1998/10/18 19:00 & A W & $\mathrm{P}$ & 2000/10/28 09:01 & S A W & $\mathrm{P}$ & 2002/05/10 10:29 & S A W & $\mathrm{P}$ \\
\hline $1998 / 10 / 23 \quad 12: 33$ & A W & $\mathrm{P}$ & $2000 / 10 / 31 \quad 16: 23$ & S A W & $\mathrm{C}$ & 2002/05/11 09:16 & S A W & $\mathrm{P}$ \\
\hline 1998/11/07 07:35 & S A W & A & $2000 / 11 / 04$ 01:30 & S A W & $\mathrm{C}$ & 2002/05/18 19:19 & A S W & $\mathrm{C}$ \\
\hline 1998/11/08 04:20 & S A W & $\mathrm{C}$ & $2000 / 11 / 2605: 00$ & A W & $\mathrm{C}$ & $2002 / 05 / 2120: 59$ & A W & A \\
\hline 1999/03/10 00:40 & A S W & $\mathrm{P}$ & 2000/11/26 11:24 & A S W & $\mathrm{C}$ & $2002 / 05 / 2310: 15$ & A S W & $\mathrm{C}$ \\
\hline 1999/05/05 14:46 & S A W & $\mathrm{C}$ & $2000 / 11 / 28$ 04:47 & S A W & $\mathrm{C}$ & $2002 / 05 / 3001: 25$ & S A W & $\mathrm{A}$ \\
\hline $1999 / 06 / 1511: 55^{*}$ & A W & $\mathrm{A}$ & 2000/12/03 03:20 & A W & $\mathrm{C}$ & $2002 / 07 / 17$ 15:26 & A S W & $\mathrm{C}$ \\
\hline 1999/06/26 02:18 & A W S & $\mathrm{C}$ & 2001/01/23 10:06 & A S W & $\mathrm{C}$ & $2002 / 07 / 1909: 32$ & A S & $\mathrm{C}$ \\
\hline $1999 / 06 / 2619: 20$ & A S W & $\mathrm{C}$ & $2001 / 01 / 3107: 22$ & A S W & $\mathrm{C}$ & $2002 / 07 / 1914: 41$ & A S W & $\mathrm{C}$ \\
\hline 1999/06/27 22:30 & A W & $\mathrm{P}$ & 2001/03/19 10:12 & S A W & $\mathrm{C}$ & $2002 / 07 / 2912: 40$ & A S W & A \\
\hline 1999/07/02 00:23 & S W A & $\mathrm{P}$ & $2001 / 03 / 27$ 17:02 & S A W & $\mathrm{C}$ & $2002 / 08 / 0122: 19$ & A S W & $\mathrm{C}$ \\
\hline $1999 / 07 / 2623: 33$ & A W & $\mathrm{P}$ & $2001 / 03 / 3021: 51$ & A W & $\mathrm{P}$ & $2002 / 08 / 18$ 18:10 & A S W & $\mathrm{A}$ \\
\hline 1999/07/30 10:21* & A W & $\mathrm{C}$ & 2001/03/31 00:14 & S A W & $\mathrm{C}$ & 2002/08/26 10:20 & S A W & $\mathrm{P}$ \\
\hline 1999/08/04 01:15 & A S W & $\mathrm{C}$ & 2001/03/31 21:40* & $\mathrm{A} \mathrm{W}$ & $\mathrm{C}$ & $2002 / 09 / 07$ 15:54 & S A W & $\mathrm{C}$ \\
\hline 1999/08/08 17:44 & A & A & 2001/04/04 14:21 & S A W & $\mathrm{C}$ & $2002 / 11 / 09$ 17:54 & A S W & $\mathrm{A}$ \\
\hline 1999/08/15 09:38 & A S W & A & $2001 / 04 / 07$ 16:45 & S A W & $\mathrm{P}$ & $2002 / 11 / 2621: 10$ & A S W & $\mathrm{C}$ \\
\hline $1999 / 08 / 22$ 22:48 & A W & A & 2001/04/08 10:23 & S A W & $\mathrm{C}$ & $2002 / 12 / 2209: 45^{*}$ & A W & $\mathrm{P}$ \\
\hline $1999 / 08 / 23$ 07:28* & A W & A & $2001 / 04 / 11$ 15:18 & S A W & $\mathrm{C}$ & $2002 / 12 / 22 \quad 12: 16$ & $\mathrm{AW}$ & $\mathrm{P}$ \\
\hline 1999/09/12 03:15 & S A W & A & 2001/04/14 01:20* & A W & $\mathrm{C}$ & $2003 / 03 / 2004: 20$ & A W & $\mathrm{P}$ \\
\hline 1999/09/15 07:15 & A S W & $\mathrm{P}$ & 2001/04/17 23:57 & S A W & $\mathrm{C}$ & 2003/04/08 00:04 & S A W & A \\
\hline 1999/09/15 19:45 & A W & A & 2001/04/28 04:28 & S A W & $\mathrm{C}$ & & & \\
\hline
\end{tabular}

Notes:

a The earliest observed timing of the Fast Forward shock passage (UT). An asterisk (*) denotes medium-quality plasma parameter jumps.

b Spacecraft that observed the shock. S: SOHO (Celias), A: ACE, and W: WIND. The order represents the temporal order of the observations. Note that all the observations are not necessarily found in the references of Table 1.

c The "quick-look" signal for the high-energy ESP. C: "Clearly" ESPs, P: "Probably" ESPs, and A: "ambiguous" ESPs. 
Table 3. Association between fast forward shocks and high-energy ESPs.

\begin{tabular}{lccc}
\hline Groups $^{\mathrm{a}}$ & Quality $^{\mathrm{b}}$ & ESPs: YES & ESPs: NO \\
\hline C+P vs. A+N & Medium+High & $42 \%(124)$ & $58 \%(171)$ \\
C+P vs. A+N & High & $44 \%(118)$ & $56 \%(153)$ \\
C vs. N & Medium+High & $38 \%(85)$ & $62 \%(137)$ \\
C vs. N & High & $40 \%(81)$ & $60 \%(121)$ \\
\hline
\end{tabular}

\begin{tabular}{l}
\multicolumn{1}{c}{ Average percentage $41 \%$} \\
\hline Notes: \\
${ }^{a}$ Quick-look ESP signal groups for "YES" and "NO" ESP signals: \\
C: "Clearly", P: "Probably", A: "Ambiguous", and N: "No" \\
b The reliability of the fast forward shock identification.
\end{tabular}

year. On the other hand, the "clearly" group reaches local minimum whereas the "probably" group reaches local maximum during that year. There is no obvious explanation for this behavior.

\section{Proton spectra at the time of shock passage}

The intensity of $\mathrm{MeV}$ protons at the time of shock passage can be used as a crude measure for the local acceleration efficiency of ICME-driven shocks (Kallenrode, 1996). To investigate the applicability of this assumption in the present sample of shocks, the shock "passage spectra" were calculated for all fast forward shocks.

In this study, the shock passage spectra were determined using the proton energy range $1.78-10.1 \mathrm{MeV}$ with eight channels. It is anticipated that the number of ESP events decreases as the highest observed particle energy of the ESP event increases. Therefore, the energies used in calculating the spectra were chosen from the low-energy end of the energy range covered by ERNE. The passage spectra were produced automatically from the ERNE proton intensity measurements at the time of the reported shock passages without concerning whether ESPs were actually observed or not. Because it would be very difficult in an automatic procedure to reliably take into account the background, the intensities were not background corrected. The motivation for this approach is that such a calculation could easily be done in realtime, and perhaps used for forecasting purposes.

The passage spectra were determined for all the fast forward shocks excluding the cases without available ERNE data (data gap or corrupted data). This yielded a total of 286 fast forward shocks. CELIAS timings were available for 159 fast forward shocks, and for the remaining 127 shocks, ACE or WIND timings, which ever was earlier, were used.

The proton intensities were integrated over $31 \mathrm{~min}$ centering at the shock passage times $(15 \mathrm{~min}+1 \mathrm{~min}+15 \mathrm{~min})$. The passage spectra were determined by fitting the data by a power law of the standard form: $I(E)=\alpha E^{\gamma}$. Here $I(E)$ is the observed intensity at energy $E, \alpha$ is the power law factor, and $\gamma$ is the spectral index.

For comparison, reference spectra were also determined. The reference spectra were calculated every day at 12:00 UT in the same way as the passage spectra.

Figure 7 presents the evolutions of the fit parameters during the study period. The panels on the left show the spectral indices, the logarithms of power law factors, and the $\chi^{2}$ values of the fits for the passage spectra. Red squares identify the shocks that are "clearly" associated with ESPs. The panels on the right show the same quantities, but for the reference spectra. To illustrate the evolution trends, second order fits are drawn for the spectral indices and the $\chi^{2}$-values, and third order fits for the logarithms of the power law factors.

There seems to be no trend in the evolution of the spectral indices, but the power law factors increase during the solar activity maximum (around 2001) compared to the minimum (around 1996). The $\chi^{2}$-values reveal that the standard power law fits the data well in the studied energy range, and there is no obvious solar cycle trend, although the passage spectra $\chi^{2}$-values seem to reach a shallow minimum during solar maximum. Comparing the passage and reference spectra, it is immediately clear that the solar cycle trend of the passage spectra power law factors just reflects the background behavior. However, the cases, which are "clearly" associated with ESPs (red squares), have larger power law factors and smaller $\chi^{2}$-values than the other cases basically during the whole study period.

Figure 8 shows the distributions of spectral indices (left) and power law factors (right). The shaded histograms represent the distributions of the whole passage spectra sample. The thin lines represent the reference distributions, which are normalized to the maximum value of the passage spectra distributions. The sub-groups of "clearly ESPs" and "no ESPs" are drawn with the thick red and thick black histograms, respectively.

The distributions of spectral indices can be described with Gaussian distributions, although the maximum of the reference distribution seems to be slightly shifted towards large spectral index values. It is evident that the distributions of the whole passage spectra sample and the reference spectra are not identical. The averages and standard deviations of the passage and reference distributions are $\left\langle\gamma_{\text {pas,all }}\right\rangle=-3.1 \pm 1.1$ and $\left\langle\gamma_{\text {ref }}\right\rangle=-2.78 \pm 0.99$, respectively. The Student's $t$-test verifies that the average passage spectrum is significantly steeper than the average reference spectrum. It is also evident that the sub-distributions "clearly ESPs" and "no ESPs" deviate from each other. The averages and standard deviations are $\left\langle\gamma_{\text {pas, clearly }}\right\rangle=-3.6 \pm 1.3$ and $\left\langle\gamma_{\text {pas,no }}\right\rangle=-2.89 \pm 0.90$, respectively. The difference is statistically significant.

As expected from Fig. 7, the distributions of the power law factors are non-Gaussian due to the solar cycle variation. Clearly, the distribution of the whole passage spectra sample includes a random component that can be represented 

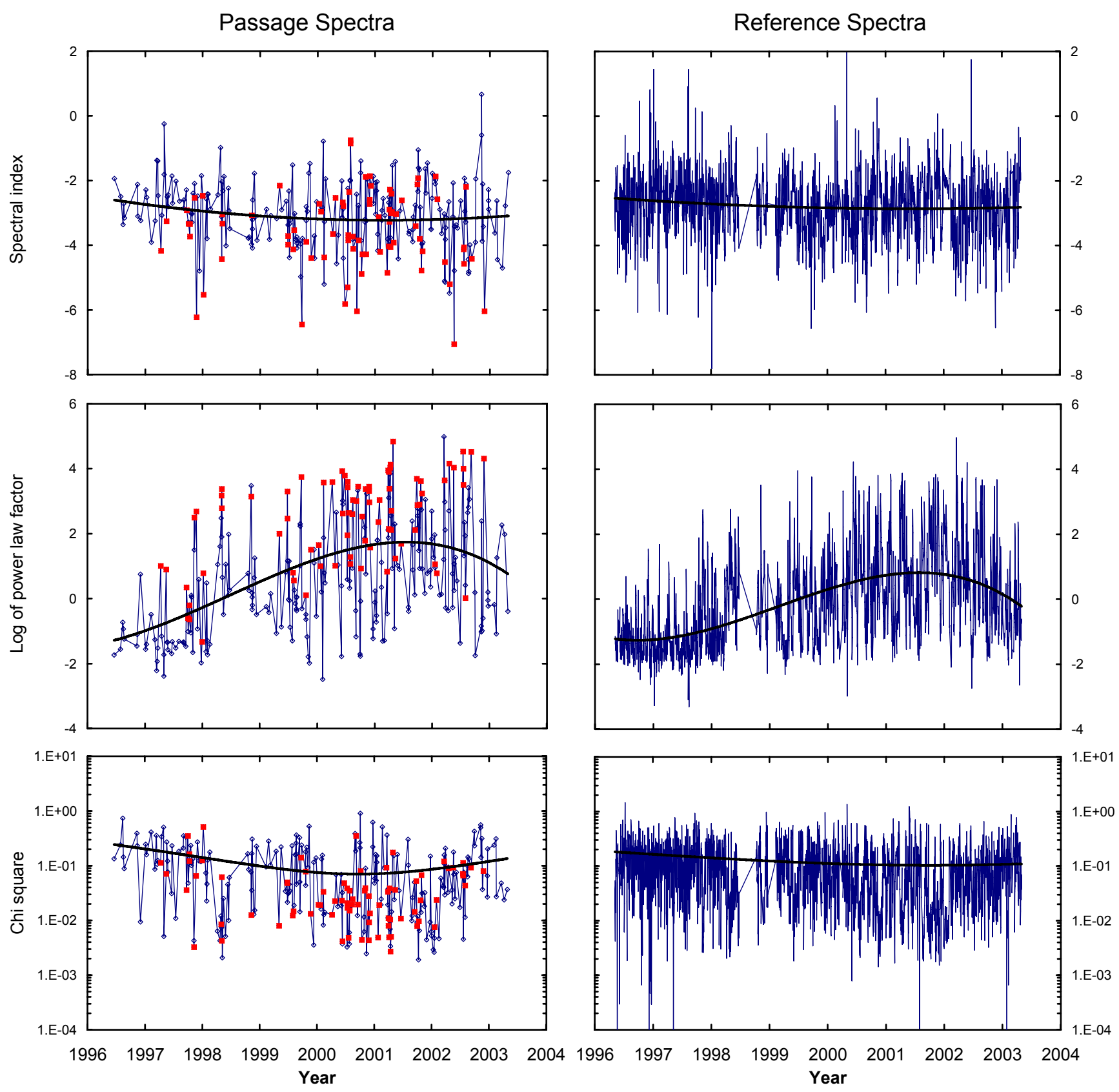

Fig. 7. The evolutions of the $1.78-10.1 \mathrm{MeV}$ proton power law fit parameters during the study period. The panels on the left show the passage spectra parameters associated with the fast forward shocks (286, ERNE data available). The spectra are 31-min averages centered on the reported shock passage times. Red squares mark the shocks that are "clearly" associated with ESPs. The panels on the right show the reference spectra parameters for comparison. The reference spectra were calculated every day at 12:00 UT in the same way as the passage spectra.

by the reference spectra. The statistically significantly deviating averages of the passage and reference distributions are $\left\langle\log \alpha_{\text {pas,all }}\right\rangle=1.0 \pm 1.8$ and $\left\langle\log \alpha_{\text {ref }}\right\rangle=-0.1 \pm 1.5$, respectively. For the "clearly ESPs" and "no ESPs" subdistributions the averages are $\left\langle\log \alpha_{\text {pas,clearly }}\right\rangle=2.4 \pm 1.5$ and $\left\langle\log \alpha_{\text {pas,no }}\right\rangle=0.1 \pm 1.5$, respectively.
With the evidence above, it is justified to say that the passage spectra of the "no ESPs" cases are nothing more than random spectra. The passage spectra of the "clearly ESPs" cases, on the other hand, are significantly different from the random spectra, which proves that those spectra clearly contain ESPs. 

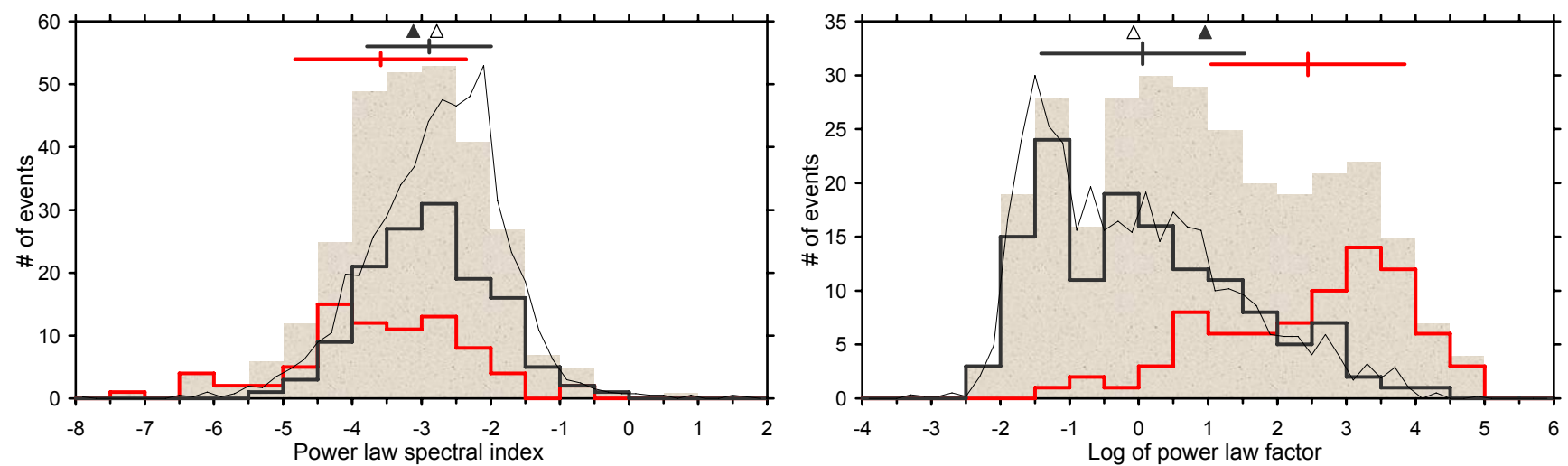

Fig. 8. The distributions of the power law parameters of the proton passage spectra. The proton spectra were derived for the 286 fast forward shock passages with integration time of $31 \mathrm{~min}$ centered on the reported shock passage times. The fits of the proton spectra were made in the energy range 1.78-10.1 MeV. The spectral indices are presented on the left panel and the logarithms of the power law factors on the right panel. The shaded areas represent the distributions of the whole passage spectra sample regardless whether ESPs were observed or not. For comparison, the normalized reference distributions are shown with the thin curves. The sub-distributions of the events with "clear ESPs" and "no ESPs" are drawn with the red and black thick lines, respectively. The averages and the standard deviations (horizontal bars) of the sub-distributions are shown in the panels. Filled [open] triangle corresponds to the average of the whole passage spectra [reference spectra] sample.

\section{Discussion and conclusions}

In the course of this investigation, a database containing several hundred IP shocks (and other solar wind structures) from 1 May 1996 to 30 April 2003, was compiled. It was found that at the distance of $1 \mathrm{AU} 77 \%$ of the interplanetary shocks could be identified as fast forward shocks, $8 \%$ as fast reverse shocks, $10 \%$ as slow forward shocks, and $5 \%$ as slow reverse shocks. The solar cycle variation was clearly seen in the yearly amount of the fast forward shocks. During May 2000April 2001 roughly four times more fast forward shocks (74) were observed than during May 1996-April 1997 (18). The trend is similar to that found by Echer et al. (2003), but the solar cycle dependence that we observed in the number of fast forward shocks is not as strong (10-fold) as reported by Echer et al. (2003). The results are not, however, entirely comparable since Echer et al. (2003) inspected years 19951996 at the activity minimum period, while our sample may be affected by the early rising phase of the cycle.

The main purpose of the present work was to survey the fast forward shock passages that were associated with highenergy $(>1.5 \mathrm{MeV})$ ESPs by using a simple classification of the ESP signatures. We also investigated proton energy spectra at the time of the shock passage irrespective of the occurrence of energetic storm particles at that time and compared them with reference spectra measured each day at noon. In addition, we exploited the relatively long study period from the minimum to well past the solar maximum activity to investigate the solar cycle dependence of the ESP occurrence with fast forward shocks.

The number of fast forward shocks during our study period for which ERNE particle data were available reached
295. However, the datasets of nine of the cases could not be used for quantitative analysis due to corrupted particle data. A qualitative "quick-look" classification of the ESP signals was achieved by making a visual inspection of the proton intensities (1.5-130 MeV, 20 energy channels). Intensity enhancements without velocity dispersion at several energy channels temporally in the vicinity of the shock passage were considered as ESP event candidates. During the study period, roughly $40 \%$ of the observed interplanetary fast forward shocks accelerated ESPs (protons) to energies greater than $1.5 \mathrm{MeV}$. Near the maximum of solar cycle 21 (August 1978-November 1979) Tsurutani and Lin (1985) found acceleration effects above $\sim 1.5 \mathrm{MeV}$ in $27 \%$ of the fast forward shocks, while Kallenrode (1996) using 3.7-13.8 MeV proton data between 1974 and 1985, thus also covering the time period studied by Tsurutani and Lin (1985), concluded that $53 \%$ of fast forward shocks had ESP signatures. In a more recent study of Lario et al. (2003) covering the time period from September 1997 to December 2001 (roughly from the minimum to the maximum of solar cycle 23) the corresponding portion was $33 \%$ for $1.9-4.8 \mathrm{MeV}$ ions. This is roughly comparable to our present result, which was obtained for an overlapping but wider time period. It should be noticed, however, that in each of these studies somewhat different energy ranges and classifications of the ESP signatures were used. As well, the applied phenomenological classifications are subjective. We also found that the highenergy ESP-effectiveness of the fast forward shocks had a solar cycle dependence. The yearly ESP-effectiveness varied from 11\%, in May 1996-April 1997 ( activity minimum), to 53\% in May 2000-April 2001 ( activity maximum). To our knowledge, a solar cycle dependence of the high-energy 
ESP-effectiveness of fast forward shocks has not been reported before. Energetic storm particle signatures associated with 191 fast forward transient shocks, from 1 February 1998 to 28 October 2003, was previously investigated by Lario et al. (2005). They classified the ESP events in six different types based on 47-68 keV ion observations (Lario et al., 2003). The occurrence and type of energetic storm particle events were not found to correlate well with any specific shock characteristics. Lario et al. (2005) suggested that the combination of all shock parameters, together with the presence or absence of an energetic seed particle population play a key role in ESP occurrence associated with fast forward shocks and their final characteristics. Tsurutani and Lin (1985) also pointed out the importance of seed particles to shock acceleration of particles in interplanetary space. Kallenrode (1996) has, however, questioned the need for preaccelerated seed particles in the production of MeV-range ESPs. Kallenrode (1996) found that 29\% of 351 transient IP shocks were associated with "pure interplanetary particle" events which presumably are ESP events without preceding SEP events. While we did not classify the particle signatures in the same way as Kallenrode (1996), we find that the ESP event on 30 December 1997 is a most clearest example of such an event (see panel d of Fig. 4). That event is intriguing because its characteristics are unique in this survey: it occurs on a quiet background (the last preceding SEP event was observed roughly ten days earlier) and the intensities start to rise only after the shock passage, which means that the particles have not at all leaked out to the upstream side of the shock.

We are not able to conclusively explain the high-energy ESP-effectiveness of fast forward interplanetary shocks or its solar cycle dependence found in the present investigation. The ESP-effectiveness of transient fast forward shocks is most probably dependent on the history of the dynamically evolving shock while propagating from the Sun to the observer, as stressed by Lario et al. (2005) and Cohen (2006). Explanation of the solar cycle dependence may be related to the relative portion of various shock drivers in different phases of the solar activity cycle. The reason may also lie in the different conditions prevailing in the interplanetary space during solar minimum and solar maximum. Near solar maximum the seed particle population in interplanetary space originating from SEP events is probably more abundant than during solar minimum conditions. In addition, conditions in IP space may be more favourable for particle acceleration/entrapment due to increased magnetic turbulence. Both these factors would enhance ESP-effectiveness of FF shocks during solar maximum. These issues will be further studied in the continuation of the present work.

In this study, we performed a quantitative analysis of the proton power law spectra at the time of the shock passage. Proton spectra were calculated for every fast forward shock passage in the energy range $1.78-10.1 \mathrm{MeV}$, irrespective of the "quick-look" ESP signal classification. It was found that the average passage spectrum for events, which had been "clearly" associated with ESPs, was significantly steeper than the average spectrum without ESPs. The average spectral indices and standard deviations were $-3.6 \pm 1.3$ and $-2.89 \pm 0.90$, respectively. van Nes et al. (1984), studying energy spectra of protons associated with interplanetary shocks at relatively low energies $(35-1600 \mathrm{keV})$ found that in general the spectrum was described by two power laws with a breakpoint near $250 \mathrm{keV}$. In cases where the spectrum could be described with a single power law over the entire energy range, they found spectral indices between -2.2 and -3.6. Kallenrode (1996) obtained spectral indices of -3.7 and -3.5 for her categories of pure interplanetary particle events and events associated with SEPs, respectively, in the proton energy range from 4 to $50 \mathrm{MeV}$. In our study, the clear ESP cases had significantly larger power law factors than the cases without ESPs. The average power law factors were $10^{(2.4 \pm 1.5)}$ and $10^{(0.1 \pm 1.5)}$, respectively. For comparison, blind reference spectra were determined every day at 12:00 UT. The spectral index of the average reference spectrum was $-2.78 \pm 0.99$, and the power law factor $10^{(-0.1 \pm 1.5)}$. These values are basically the same as for the fast forward shock passages, which in our study were considered to lack high-energy ESPs. Therefore, the particle intensities during the fast forward shock passages that were given "no ESPs" signal in the visual inspection, do not differ from the average spectrum at a random time. Thus, the validity of the "no ESPs" and the "clearly ESPs" quick-look signals is confirmed. This analysis can not, however, confirm the nature of a single case, and did not take into account the effects of background on the results or the possible time difference between the shock passage time and the intensity peak.

In order to get elaborate information of the high-energy ESPs, the events have to be analyzed case by case. A complete observational view may be achieved only if the details of the ESP events are studied against the details of the interplanetary shocks causing them, against the details of the magnetic structures driving the shocks, and against the details of the interplanetary environment conditions. The present survey will be used as the groundwork for a more detailed investigation to better understand the conditions and processes of particle acceleration in interplanetary shocks.

Acknowledgements. This work was partly supported by the Academy of Finland under the decision \#104329. We acknowledge ACE team, Berdichevsky et al., CELIAS shockspotter team, D. Larson, and J. Kasper for the use of their interplanetary shock catalogs. We thank Coordinated Data Analysis Web (CDAWeb) for making easy access to ACE/MFI (N. Ness) data, ACE/SWEPAM (D. J. McComas) data, WIND/MFI (R. P. Lepping) data, WIND/3DP (R. P. Lin) data, and WIND/SWE (K. W. Ogilvie) data. K. H.-H. wishes to thank the Väisälä Foundation of the Finnish Academy of Science and Letters for a travel grant. We thank the two referees for evaluating this work. SOHO is a project of international cooperation between ESA and NASA. 
Topical Editor R. Forsyth thanks B. Heber and O. Malandraki for their help in evaluating this paper.

\section{References}

Berdichevsky, D. B., Szabo, A., Lepping, R. P., Viñas, A. F., and Mariani, F.: Interplanetary fast shocks and associated drivers observed through the 23rd solar minimum by Wind over its first 2.5 years, J. Geophys. Res., 105, 27289-27314, 2000.

Berdichevsky, D. B., Szabo, A., Lepping, R. P., Viñas, A. F., and Mariani, F.: Erratum: "Interplanetary fast shocks and associated drivers observed through the 23rd solar minimum by Wind over its first 2.5 years", J. Geophys. Res., 106, 25133-25134, 2001.

Bryant, D. A., Cline, T. L., Desai, U. D., and McDonald, F. B.: Explorer 12 Observations of Solar Cosmic Rays and Energetic Storm Particles after the Solar Flare of September 28, 1961, J. Geophys. Res., 67, 4983-5000, 1962.

Cane, H. V.: The Structure and Evolution of Interplanetary Shocks and the Relevance for Particle Acceleration, Nucl. Phys. B-Proc. Sup., 39, 35-44, 1995.

Cane, H. V. and Richardson, I. G.: Interplanetary coronal mass ejections in the near-Earth solar wind during 1996-2002, J. Geophys. Res., 108, 1156-1168, 2003.

Channok, C., Ruffolo, D., Desai, M. I., and Mason, G. M.: FiniteTime Shock Acceleration of Energetic Storm Particles, Astrophys. J., 633, L53-L56, 2005.

Cohen, C. M. S.: Observations of Energetic Storm Particles: An Overview, Solar Eruptions and Energetic Particles, ed. N. Gopalswamy, R. Mewaldt, and J. Torsti, Geophysical Monograph, 165, 275-282, 2006

Desai, M. I., Mason, G. M., Wiedenbeck, M. E., Cohen, C. M. S., Mazur, J. E., Dwyer, J. R., Gold, R. E., Krimigis, S. M., Hu, Q., Smith, C. W., and Skoug, R. M.: Spectral Properties of Heavy Ions Associated with the Passage of Interplanetary Shocks at 1 AU, Astrophys. J., 611, 1156-1174, 2004.

Echer, E., Gonzalez, W. D., Vieira, L. E. A., Dal Lago, A., Guarnieri, F. L., Prestes, A., Gonzales, A. L. C., and Schuch, N. J.: Interplanetary Shock Parameters during Solar Activity Maximum (2000) and Minimum (1995-1996), Brazilian Jour. Phys., 33, 115-122, 2003

Ho, G. C., Lario, D., Decker, R. B., Roelof, E. C., Desai, M., and Smith, C. W.: Energetic Electrons Associated with Transient Interplanetary Shocks: Evidence for Weak Interaction, in: International Cosmic Ray Conference, pp. 3689-3692, 2003.

Hovestadt, D., Hilchenbach, M., Bürgi, A., Klecker, B., Laeverenz, P., Scholer, M., Grünwaldt, H., Axford, W. I., Livi, S., Marsch, E., Wilken, B., Winterhoff, H. P., Ipavich, F. M., Bedini, P., Coplan, M. A., Galvin, A. B., Gloeckler, G., Bochsler, P., Balsiger, H., Fischer, J., Geiss, J., Kallenbach, R., Wurz, P., Reiche, K.-U., Gliem, F., Judge, D. L., Ogawa, H. S., Hsieh, K. C., Möbius, E., Lee, M. A., Managadze, G. G., Verigin, M. I., and Neugebauer, M.: CELIAS - Charge, Element and Isotope Analysis System for SOHO, Sol. Phys., 162, 441-481, 1995.

Howard, T. A. and Tappin, S. J.: Statistical survey of earthbound interplanetary shocks, associated coronal mass ejections and their space weather consequences, Astron. Astrophys., 440, 373-383, 2005.

Jian, L., Russell, C. T., Luhmann, J. G., and Skoug, R. M.: Properties of Stream Interactions at One AU During 1995-2004, Sol.
Phys., 239, 337-392, 2006.

Kallenrode, M.-B.: A statistical survey of 5-MeV proton events at transient interplanetary shocks, J. Geophys. Res., 101, 24393 24410, 1996

Kallenrode, M.-B.: The temporal and spatial development of $\mathrm{MeV}$ proton acceleration at interplanetary shocks, J. Geophys. Res., 102, 22347-22364, 1997.

Lario, D., Ho, G. C., Decker, R. B., Roelof, E. C., Desai, M. I., and Smith, C. W.: ACE Observations of Energetic Particles Associated with Transient Interplanetary Shocks, in: AIP Conf. Proc. 679: Solar Wind Ten, edited by: Velli, M., Bruno, R., Malara, F., and Bucci, B., pp. 640-643, 2003.

Lario, D., Hu, Q., Ho, G. C., et al.: Statistical Properties of Fast Forward Transient Interplanetary Shocks and Associated Energetic Particle Events: ACE Observations, in: Solar Wind 11/SOHO 16, Connecting Sun and Heliosphere, vol. 592 of ESA Special Publication, pp. 13.1-13.6, 2005.

Lepping, R. P., Acũna, M. H., Burlaga, L. F., Farrell, W. M., Slavin, J. A., Schatten, K. H., Mariani, F., Ness, N. F., Neubauer, F. M., Whang, Y. C., Byrnes, J. B., Kennon, R. S., Panetta, P. V. Scheifele, J., and Worley, E. M.: The Wind Magnetic Field Investigation, Space Sci. Rev., 71, 207-229, 1995.

Lin, R. P., Anderson, K. A., Ashford, S., Carlson, C., Curtis, D., Ergun, R., Larson, D., McFadden, J., McCarthy, M., Parks, G. K., Rème, H., Bosqued, J. M., Coutelier, J., Cotin, F., D’Uston, C., Wenzel, K.-P., Sanderson, T. R., Henrion, J., Ronnet, J. C., and Paschmann, G.: A Three-Dimensional Plasma and Energetic Particle Investigation for the Wind Spacecraft, Space Sci. Rev., 71 125-153, 1995.

Lindsay, G. M., Russell, C. T., Luhmann, J. G., and Gazis, P.: On the sources of interplanetary shocks at $0.72 \mathrm{AU}, \mathrm{J}$. Geophys. Res., 99, 11-17, 1994.

Luhmann, J. G. and Mann, A.: Relative fluxes of shock and prompt peaks in SEP event time profiles, Adv. Space Res., 39, 1882 1889, 2007.

McComas, D. J., Bame, S. J., Barker, P., Feldman, W. C., Phillips, J. L., Riley, P., and Griffee, J. W.: Solar Wind Electron Proton Alpha Monitor (SWEPAM) for the Advanced Composition Explorer, Space Sci. Rev., 86, 563-612, 1998.

Meyer, J., Wibberenz, G., and Kallenrode, M.-B.: Timedevelopment of proton energy spectra in solar energetic particle events, Adv. Space Res., 13, 363-366, 1993.

Ogilvie, K. W., Chornay, D. J., Fritzenreiter, R. J., Hunsaker, F., Keller, J., Lobell, J., Miller, G., Scudder, J. D., Sittler, Jr., E. C., Torbert, R. B., Bodet, D., Needell, G., Lazarus, A. J., Steinberg, J. T., Tappan, J. H., Mavretic, A., and Gergin, E.: SWE, A Comprehensive Plasma Instrument for the Wind Spacecraft, Space Sci. Rev., 71, 55-77, 1995.

Rao, U. R., McCracken, K. G., and Bukata, R. B.: Cosmic ray propagation processes 2 . The energetic storm-particle event , J. Geophys. Res., 62, 4325-4341, 1967.

Reames, D. V.: Particle acceleration at the Sun and in the heliosphere, Space Sci. Rev., 90, 413-491, 1999.

Richardson, I. G., Cliver, E. W., and Cane, H. V.: Sources of geomagnetic storms for solar minimum and maximum conditions during 1972-2000, Geophys. Res. Lett., 28, 2569-2572, 2001.

Smith, C. W., L'Heureux, J., Ness, N. F., Acuña, M. H., Burlaga, L. F., and Scheifele, J.: The ACE Magnetic Fields Experiment, Space Sci. Rev., 86, 613-632, 1998. 
Torsti, J., Valtonen, E., Lumme, M., Peltonen, P., Eronen, T., Louhola, M., Riihonen, E., Schultz, G., Teittinen, M., Ahola, K., Holmlund, C., Kelha, V., Leppala, K., Ruuska, P., and Strommer, E.: Energetic Particle Experiment ERNE, Sol. Phys., 162, 505-531, 1995.

Tsurutani, B. T. and Lin, R. P.: Acceleration of greater than $47 \mathrm{keV}$ ions and greater than $2 \mathrm{keV}$ electrons by interplanetary shocks at 1 AU, J. Geophys. Res., 90, 1-11, 1985.

Valtonen, E., Laitinen, T., and Huttunen-Heikinmaa, K.: Energetic particle signatures of geoeffective coronal mass ejections, Adv. Space Res., 36, 2295-2302, 2005.

van Nes, P., Reinhard, R., Sanderson, T. R., Wenzel, K.-P., and Zwickl, R. D.: The energy spectrum of $35-$ to $1600-\mathrm{keV}$ protons associated with interplanetary shocks, J. Geophys. Res., 89, 2122-2132, 1984.
Zhang, J., Richardson, I. G., Webb, D. F., Gopalswamy, N., Huttunen, E., Kasper, J., Nitta, N. V., Poomvises, W., Thompson, B. J., Wu, C.-C., Yashiro, S., and Zhukov, A. N.: Correction to "Solar and interplanetary sources of major geomagnetic storms (Dst=-100 nT) during 1996-2005”, J. Geophys. Res., 112, 12103-12107, 2007a.

Zhang, J., Richardson, I. G., Webb, D. F., Gopalswamy, N., Huttunen, E., Kasper, J. C., Nitta, N. V., Poomvises, W., Thompson, B. J., Wu, C.-C., Yashiro, S., and Zhukov, A. N.: Solar and interplanetary sources of major geomagnetic storms (Dst $=-100 \mathrm{nT})$ during 1996-2005, J. Geophys. Res., 112, 10102-10120, $2007 \mathrm{~b}$. 